\title{
UCRL-PROC-206303
}

LAWRENCE LIVERMORE N A TIO N A L LABORATORY

\section{Spectroscopic Imaging \\ Diagnostics for Burning Plasma Experiments}

D. Stutman, M . Finkenthal, G. Suliman, K. Tritz, L. Delgado-Aparicio, R. Kaita, D. Johnson, V. Soukhanovskii, M. J. May

August 30, 2004

15th Topical Conference on High Temperature Plasma Diagnostics San Diego, CA, United States April 21, 2004 through April 25, 2004 
This document was prepared as an account of work sponsored by an agency of the United States Government. Neither the United States Government nor the University of California nor any of their employees, makes any warranty, express or implied, or assumes any legal liability or responsibility for the accuracy, completeness, or usefulness of any information, apparatus, product, or process disclosed, or represents that its use would not infringe privately owned rights. Reference herein to any specific commercial product, process, or service by trade name, trademark, manufacturer, or otherwise, does not necessarily constitute or imply its endorsement, recommendation, or favoring by the United States Government or the University of California. The views and opinions of authors expressed herein do not necessarily state or reflect those of the United States Government or the University of California, and shall not be used for advertising or product endorsement purposes. 


\section{Spectroscopic Imaging Diagnostics for Burning Plasma Experiments}

D. Stutman, M. Finkenthal, G. Suliman ${ }^{1}$, K. Tritz, L. Delgado-Aparicio, Dept. of Physics and Astronomy, Johns Hopkins University, Baltimore, Maryland 21218

R. Kaita, D. Johnson

Plasma Physics Laboratory, Princeton University, Princeton, New Jersey 08543

V. Soukhanovskii, M.J. May

Lawrence Livermore National Laboratory, P.O. Box 5508, Livermore, California 94551

(Presented at the $15^{\text {th }}$ Topical Conference on High Temperature Plasma Diagnostic, 21 April 2004)

Spectroscopic imaging of plasma emission profiles from a few eV to tens of keV enables basic diagnostics in present day tokamaks. For the more difficult burning plasma conditions, new light extraction and detection techniques, as well as new instrument designs need to be investigated. As an alternative to light extraction with reflective optics, we discuss normal incidence, transmissive-diffractive optics (e.g., transmission gratings), which might withstand plasma exposure with less degradation of optical properties. Metallic multilayer reflectors are also of interest for light extraction. Although a shift of the diffraction peak might occur, instrument designs that accommodate such shifts are possible. As imaging detectors we consider 'optical' arrays based on conversion of the short-wavelength light into visible light followed by transport of the visible signal with hollow light-guides. The proposed approaches to light extraction and detection could enable new and radiation resistant diagnostics.

\footnotetext{
${ }^{1}$ Permanent address: "Horia Hulubei" National Institute of Physics and Nuclear Engineering, Department of Nuclear Physics, Bucharest, Romania
} 


\section{INTRODUCTION}

The diagnostic of 'burning plasma' experiments in which deuterium-tritium fusion reactions are the main heating source, will pose a major challenge. Although at present the choices for a next-step device in the US fusion program are still open, such an experiment is deemed essential for the progress of fusion research.

Among the basic measurements, spectroscopic imaging of plasma emission profiles in the energy range from a few $\mathrm{eV}$ to tens of $\mathrm{keV}$ (visible to $\mathrm{X}$-rays) can play an essential role for machine control and operation, as well as for plasma performance evaluation and physics studies [1]. As one will approach the burning plasma environment, these measurements will however be considerably more difficult, due to intense plasma and nuclear radiation, as well as to long plasma exposure. As an example, the 'first mirrors' directly viewing the International Thermonuclear Reactor (ITER) plasma will be exposed for hundreds of hours each year to energetic neutron and gamma fluxes of $\approx 10^{12} \mathrm{~cm}^{-2} \mathrm{~s}^{-1}$, charge-exchange (CX) neutral fluxes of $10^{13} \mathrm{~cm}^{-2} \mathrm{~s}^{-1}$ and heat fluxes of $\approx 1.5 \mathrm{~kW} / \mathrm{m}^{2}$ [1]. In addition, the optical elements in proximity to the burning plasma can be serviced or replaced only remotely, due to activation of the adjacent structural materials.

The present designs for burning plasma spectroscopic systems extrapolate the large tokamak experience. Typically plasma light is extracted using a metallic first mirror and then sent to a remote spectrometric system using secondary mirrors arranged in a labyrinth path in the radiation shield. There are several difficulties with this approach. First, the primary mirrors are subjected to intense sputtering and coating by neutral atoms escaping the plasma [2]. This causes mirror reflectivity loss and changes in the spectral and polarizing properties, which can severely impact critical diagnostics, such as for instance active beam spectroscopy [3,4]. Second, maintaining the accurate optical alignment over long ( $\geq 10 \mathrm{~m}$ in ITER) multiply folded beam paths in the harsh burning plasma environment is also difficult. Finally, using the remote spectrometry approach, wide-angle measurements of the plasma profiles often require using multiple, expensive beam-lines [1].

It is therefore important to investigate also new ideas and tools for burning plasma spectroscopic diagnostic. In particular, spectroscopic imaging instruments that could function in closer proximity to the burning plasma could provide more cost effective and robust techniques for some of the critical measurements, such as for instance impurity content in the divertor and main plasma. In the present paper we focus on new elements for the extraction and detection of XUV light (ultrasoft X-ray to VUV, or the few $\AA$ to $\approx 2000 \AA$ range) in the burning plasma environment. Some of these elements can also be of interest also for visible light diagnostics, such as active beam spectroscopy. Many of the discussed devices are made possible through recent advances in nanofabrication technology. 
The structure of the paper is as follows. Section II discusses short wavelength light extraction using diffractive optical elements such as transmission gratings. Section III focuses on light extraction with multilayer mirrors, while Section IV discusses possible approaches for invessel detection of XUV light from the burning plasma. Throughout the paper we use ITER radiation numbers for exemplification purposes, since at present these provide the best description of the burning plasma environment.

\section{XUV LIGHT EXTRACTION WITH DIFFRACTIVE OPTICAL ELEMENTS}

A first idea we discuss is using normal incidence, transmissive-diffractive optical elements as an alternative to the grazing incidence mirrors conventionally used for short wavelength light extraction. The basic principle is to use diffraction off a freestanding metallic structure such as a transmission grating or a Fresnel zone plate, in order to deflect a usable portion of the incident light out of the direct plasma view and into a particle and radiation shielded measurement region.

The advantage offered by this approach might be that the active light-deflecting element is not an extended material surface, but an array of thin metallic wires. Thus even under heavy neutron, gamma and particle bombardment, and even with some plasma impurity deposition, there is a better chance that such a device will withstand the direct plasma view without significant altering its optical properties. Furthermore, even if slow efficiency degradation would occur, it would be easier to use interchangeable or movable gratings in the beam path, since at normal incidence the sensitivity to misalignment is low.

Transmission gratings (TGs) have been used for quite some time as soft X-ray dispersive elements in the spectroscopy of laboratory and astrophysical plasmas [5,6]. Freestanding metallic gratings with up to 10,000 lines/mm can nowadays be produced by electron beam lithography in a sub-micron thick substrate [5].

Using such gratings we recently tested on the NSTX and CDX-U tokamaks simple and compact imaging spectrometers for the ultrasoft X-ray (few $\AA$ to $\approx 300 \AA$ ) range. These are in essence spectrally resolved pinhole cameras, in which two narrow slits and a normal incidence grating disperse and image the light onto a two-dimensional detector. As illustrated by the CDXU spectrum in Figure 1 , spectral resolution $\Delta \lambda / \lambda \approx 0.03-0.06$ and spatial resolution $\Delta \mathrm{r} / \mathrm{a} \approx 0.05$ 0.10 (a, device minor radius) were obtained in this simple setup [7].

Similar instruments could be of interest for the spectroscopy of the burning plasma. In particular, these non-focusing devices would be well suited for survey spectrometry of the divertor region, where bright XUV line emission $\left(10^{15-} 10^{17}\right.$ photons $\left.\mathrm{cm}^{2} \mathrm{sr}^{-1} \mathrm{~s}^{-1}\right)$ can be expected from the cool and dense plasma. Multi-chordal XUV spectroscopy will be essential for the burning plasma divertor, due to the likely use of high- $Z$ cooling gases or first wall components. Two-dimensional measurements will also be important in the divertor due to the complex plasma 
shape. Building such a diagnostic using multiple conventional spectrometers and beam lines would be costly and difficult [8].

The possible layout of a TG spectrometer for the burning plasma divertor is shown in Figure 2. Assuming the ITER geometry, adequate spatial coverage could be obtained by placing the instrument a few meters away from the divertor. A second instrument vertically viewing the divertor could be used for tomographic reconstruction of the local emissivity. As sketched for the second device, at VUV wavelengths the light could be further folded or focused after being diffracted by the grating, using for instance broad band SiC mirrors. Finally, high throughput polychromators based on grid-collimator, transmission gratings and focusing mirrors can be envisioned along the lines discussed in Section III.

To obtain spectral and spatial resolution as above, the plasma is viewed through narrow entrance and imaging slits (e.g., $120 \mu \mathrm{m}$ x $6 \mathrm{~cm}$ and $120 \mu \mathrm{m}$ x $0.6 \mathrm{~cm}$, respectively), spaced 10 $\mathrm{cm}$ apart and positioned behind a thick radiation shield. In addition to collimating the incident light, the slits serve also to drastically reduce the amount of sputtering and deposition on the TG.

For the instrument viewing only the divertor plasma the energy of the escaping neutrals will be too low to sputter a high-Z grating [2]. For the device viewing the divertor through the main plasma some sputtering will occur. Considering a grating made of high- $Z$ refractory metal such as $\mathrm{W}$ or Ta, assuming ITER first wall conditions $\left(\approx 210^{15} \mathrm{~cm}^{-2} \mathrm{~s}^{-1}\right.$ energetic $\mathrm{CX}$ atoms $)$, and using effective sputtering yields predicted for ITER in Ref. 9, one obtains that the $\approx 510^{-6} \mathrm{~cm}^{2} \mathrm{sr}$ throughput of the collimator limits the grating sputtering to several $\AA$ per ITER operation year $(\approx 400$ 'burn' hours).

More significant in the divertor could be deposition effects. Recent ITER calculations predict that the net $\mathrm{C}$ deposition rate would range from $\approx 10^{14}$ atoms $\mathrm{cm}^{-2} \mathrm{~s}^{-1}$ near the main plasma, to $\approx 210^{15}$ atoms cm $\mathrm{c}^{-2} \mathrm{~s}^{-1}$ near the outer divertor leg [10]. Assuming these rates, the $\mathrm{C}$ coating on the grating viewing the divertor would reach $\approx 600 \AA$ per ITER operation year. It is likely that such a thin low-Z overcoat on a high-Z grating will not significantly affect its diffractive properties. In addition, since as above mentioned the sensitivity to misalignment at normal incidence is low, one could 'refresh' the $\approx 120 \mu$ wide grating area exposed to the plasma by laterally translating the grating.

The above devices would require transmission gratings operating also in the VUV range. While such gratings are not commonly used for VUV spectroscopy, where less costly reflection gratings are preferred, VUV instruments based on larger period transmission gratings (500-2500 $1 / \mathrm{mm}$ ) have nevertheless been demonstrated [11,12].

Today's advances in nanofabrication would make possible optimizing the grating performance through shaping of the grating bars and choice of materials. For instance, the spectral resolution of the TG imaging spectrometer is in the first order [5]:

$$
\Delta \lambda / \lambda \approx 2(\mathrm{w} / \mathrm{L})(\mathrm{d} / \lambda)
$$


where $\mathrm{w}$ and $\mathrm{L}$ are the collimator width and length, and $\mathrm{d}$ is the grating period. The device in Figure 2 would have for instance $\Delta \lambda \approx 12 \AA$ at $600 \AA$, when using a d=5000 $⿱$ grating. This would be adequate for VUV survey spectrometry of the divertor plasma, where the spectrum is typically dominated by strong resonance transitions from low charge states of low-Z impurities, spaced some tens of $\AA$ apart (e.g., C III $2 s^{2}-2 s 2 p$ at $\approx 977 \AA$ and O VI $2 s-2 p$ at $\approx 1032 \AA$ ). It might however be possible to increase the resolution of the TG instrument by deeply shaping the grating bars and preferentially channeling the radiation in high diffraction orders. This effect has been observed since the early days of TG research and was attributed to internal reflection on the grating bars [13].

Neglecting phase effects the diffraction efficiency of a transmission grating is [14]:

$$
\frac{\mathrm{I}^{\mathrm{m}}}{\mathrm{I}^{0}}(\lambda) \approx \mathrm{F}\left[\frac{\sin \left(\mathrm{m} \pi \frac{\mathrm{a}}{\mathrm{d}}\right)}{\mathrm{m} \pi}\right]^{2}
$$

with $\mathrm{m}$ diffraction order, $\mathrm{d}$ grating period, $a$ gap between grating wires, and $\mathrm{F}$ fraction of grating open area (outside the support structure). With open area fraction up to $\approx 65 \%$ the TG efficiency can thus reach several percent in the first order [6]. Assuming for instance a ten chord TG spectrometer having $120 \mu \mathrm{m}$ wide by $0.6 \mathrm{~cm}$ high detection 'pixels' located at $\approx 10 \mathrm{~cm}$ behind the grating, one obtains for the divertor range of XUV brightness an incident power of $\approx$ 0.2-20 nW per pixel, at a wavelength of $400 \AA$ for instance. Detection possibilities for these signal levels are discussed in section IV. A TG/focusing mirror polychromator would achieve much stronger signals per detection channel.

Other diffractive optical elements of interest could be Fresnel zone plates. In addition to spectrally dispersing the incoming light, these devices also focus radiation from multi-keV to visible light $[15,16]$. For instance, arrays of 'photon sieve' metallic lenses are currently being investigated for visible light imaging in harsh environments [16]. Such devices could conceivably be also used as 'first lenses' for light extraction and focusing in the burning plasma environment. Similarly, micro-patterned Fresnel mirrors which change light direction by diffraction rather than reflection could be of interest as replacement for conventional mirrors [17].

In conclusion, freestanding diffractive optical elements in collimated optical designs might withstand exposure to the burning plasma with possibly less degradation of their light collecting and polarizing properties than reflective 'first mirrors'. 


\section{USXR LIGHT EXTRACTION WITH MULTI-LAYER MIRORS}

Other potentially important tools for XUV light extraction from the burning plasma could be synthetic multilayer mirrors (MLM). These are high throughput devices that deflect and disperse USXR light through Bragg diffraction [18]. In recent years the multilayer mirrors found more and more applications as USXR light extractors in tokamaks. The instruments using them range from single chord polychromators, to multi-chordal arrays of monochromators, and to 2-D imaging devices [19-24].

A class of multilayer based devices that could have strong potential for burning plasma diagnostics are the mirror monochromator arrays. [20-22]. These high throughput devices can image with high spectral ( $\geq 1.5 \AA$ ), spatial $(\geq 2 \mathrm{~cm}$ ) and temporal $(\geq 2 \mu \mathrm{s})$ resolution the USXR line emission from intrinsic or injected impurities, enabling measurements of the impurity density, transport and even MHD activity. As an illustration, Figure 3 shows the emission of the C VI Ly $y_{\alpha}$ shell in a beam heated NSTX plasma, measured by a 16 chord mirror array equipped with photodiode detection [20]. In addition, to a continuos measurement of the C VI profile of interest for transport assessment, the high throughput of the system enable obtaining the mode structure of MHD perturbations localized in the C VI shell. Similar measurements were performed on the CDX-U tokamak using a MLM array for the Li III Ly $\mathrm{y}_{\alpha}$ emission at $135 \AA$ A [21].

A mirror polychromator using grid-collimators for increased throughput and resolution and microchannel plates for increased sensitivity was also prototyped on LHD for transport measurements using the faint beam charge-exchange (CX) emission from tracer embedded (TESPEL) pellets [22]. The layout of the device together with illustrative traces of injected $\mathrm{Mg}$ $\mathrm{H}_{\alpha}$ emission $(\lambda \approx 45.5 \AA)$ is shown in Figure 4. As seen, good signal-to-noise and background rejection can be obtained with this design even for faint USXR transitions.

In-vessel arrays of grid-collimator/mirror monochromators resembling that in Figure 4 might enable measuring the low and high-Z impurity line emission (possibly including the helium ash) in the edge and core of the burning plasma. Both electron collision excited and beam CX excited transitions are of interest. For instance, the $\mathrm{Ly}_{\alpha}$ or $\mathrm{H}_{\alpha}$ USXR lines of low- $\mathrm{Z}$ hydrogen-like ions have orders of magnitude higher CX excitation rates than the high-n visible lines typically used in active charge exchange spectroscopy [22, 25]. This could be important in burning plasma conditions, where due to limited beam penetration and intense bremstrahlung emission the signal-to background ratio of visible light $C X$ transitions is very poor (e.g., $\geq 1 / 100$ for the C VI n=7-8 transition in the ITER core, [25]).

A possible layout for the 'unit-cell' of a mirror monochromator array for the burning plasma is shown in Figure 5. The mirror is protected from direct plasma effects and from the background light by the combination of a grid-collimator and a thin metallic filter. The use of grid-collimators in XUV instrumentation is discussed in Ref. 26. These enable obtaining in a 
compact layout spatial resolution of a few $\mathrm{cm}$ at several meters distance, together with a few percent spectral resolution and high optical throughput [22]. A difference from the device in Figure 4 is that a focusing rather than planar multilayer would be used in the burning plasma instrument, in order to increase the ratio between the useful photon flux and the nuclear background measured by the detector (see Section IV).

The effects of sputtering and deposition on the grid-collimator/MLM instrument can be estimated as above. Considering the geometry in Figure 4 (e.g., $0.1 \times 0.1 \times 10 \mathrm{~cm}$ collimator channels having $\approx 10^{-6} \mathrm{~cm}^{2}$ sr throughput each) and assuming as above ITER first wall conditions, the sputtering rate for a common XUV filter material such as Be will be around $15 \AA$ per operation year. Since the typical filter thickness is a few $\mathrm{k} \AA$, the effect is negligible. Similarly, assuming the main plasma deposition rate $\left(\approx 10^{14} \mathrm{~cm}^{-2} \mathrm{~s}^{-1}\right)$ the carbon coating will also be minimal ( $\approx 5 \AA$ 施ar).

The overall throughput of a grid-collimator is nevertheless high, since the combined area of the collimating channels can reach $\approx 70 \%$ of the cross section [26]. For instance, assuming a $3 \times 3 \mathrm{~cm}$ cross section the device in Figure 4 can have $\approx 510^{-4} \mathrm{~cm}^{2} \mathrm{sr}$ geometrical throughput. Further on, high performance multilayers typically have $\geq 20 \%$ reflectivity, while the long wavelength blocking filters can be optimized for $\approx 20 \%$ transmission [20-22]. The brightness of low-n USXR transitions from the main plasma may be expected to span the $\approx 10^{13-} 10^{15}$ photons $\mathrm{cm}^{-2} \mathrm{sr}^{-1} \mathrm{~s}^{-1}$ range (with the lower end representing beam excited transitions). Assuming then that the spherical mirror would focus the diffracted photon flux on a $3 \times 3 \mathrm{~mm}$ detector, the device in Figure 3 would produce $\approx 10-1000 \mathrm{nW}$ signals, at $30 \AA$ wavelength for instance.

In addition to resisting exposure to the burning plasma, the in-vessel mirrors need to resist also the intense neutron and gamma irradiation. One can presume for instance that the mirrors in the above monochromator arrays will be exposed to neutron fluences comparable to those at the ITER 'first mirror' location $\left(\leq 10^{19} \mathrm{~cm}^{-2},[1]\right)$. Our earlier assessment of the radiation hardness of the multilayers shows that even at such high fluence the reflectivity is maintained for some types of mirrors. For instance, $\mathrm{W} / \mathrm{B}_{4} \mathrm{C}$ mirrors exhibited only a few percent decrease in their peak reflectivity after being irradiated with $\approx 1.110^{19} \mathrm{~cm}^{-2}$ neutrons of $1-2 \mathrm{MeV}$ characteristic energy [27].

While maintaining good reflectivity, a small shift $(\approx 2 \%)$ in the wavelength of the Bragg peak was nevertheless observed for the irradiated $\mathrm{W} / \mathrm{B}_{4} \mathrm{C}$ mirrors [27]. Similar conclusions have been obtained in other irradiation tests [28]. Based on such observations the use of multilayers is presently considered only for remote instrumentation on ITER $[1,28]$.

As we earlier noted however, the above irradiation experiments have been performed at high temperature, due to nuclear heating of the mirror substrate and insufficient cooling. This left open the question if the observed Bragg peak shift was a thermal or a neutron damage effect. Our estimates were that the thermal effects are dominant [27]. Irradiation tests of cooled, or preannealed mirrors are therefore needed for a conclusive resolution of this issue. 
Even if a small shift of the Bragg peak would occur in time, we observe that optical schemes that can compensate for such shifts are in any case possible. A simple example is illustrated in Figure 6, where there the parallel beam from the grid-collimator is incident on a mirror having curvature radius of approximately 10 times the beam diameter. As shown by the USXR ray tracing calculations, even if the mirror d-spacing varies by a few percent, a nearly constant fraction of the incident photons is focused on the detector, since the region of peak reflectivity also 'shifts' on the mirror surface. Using mirrors with laterally graded d-spacing is another option, which would reduce the curvature requirements.

Transient thermal effects on the mirror d-spacing are more difficult to quantify. It is likely however that multilayers functioning in closer proximity to the burning plasma would require temperature control in order to maintain a constant $d$-spacing and reflectivity.

In conclusion, in conjunction with protective collimators and filters the multilayer mirrors might also offer useful possibilities for in-vessel light extraction from the burning plasma.

\section{IN-VESSEL XUV LIGHT DETECTION, SIGNAL AMPLIFICATION AND TRANSPORT}

\section{IV.1 XUV light detection}

The above instruments use diffractive elements to extract XUV light in closer proximity (few meters) to the burning plasma. The extracting element views the plasma through a narrow collimator in the primary radiation shield (e.g., the tritium breeding blanket in ITER), while deflecting the useful radiation towards a protected detector. Even behind this shield the nuclear background will be however quite high, requiring new approaches to in-vessel light detection.

A detector considered for in-vessel energy integrated X-ray measurements in ITER is the Vacuum Photodiode (VPD) [29]. This is in essence a high- $Z$ metal surface that converts the incoming X-ray flux into photoelectrons, which after escaping into the vacuum are collected at an anode and detected by a remote current preamplifier. While this approach might be of interest for the $\geq \mu \mathrm{W}$ signals expected from direct X-ray detection, even here the transport of small electrical currents near the burning plasma remains a serious issue. The difficulties range from Radiation Induced Electromagnetic Forces (RIEMF) in the electrical circuits [1], to electromagnetic pick-up, and to the large capacitance presented by a long cable to the current preamplifier. It is therefore likely that for the $\geq \mathrm{nW}$ signals estimated for our XUV devices the electronic detection approach is not useful.

One concept we study and which may enable more robust in-vessel XUV detectors is the 'optical array' discussed in Ref 30. In its burning plasma version it would consists of an efficient and radiation resistant XUV to visible light converter, followed by radiation hard visible light 
wave guides which transport the light signal outside the vacuum. An intermediate visible light amplification stage could be used to boost the signal before transport with the wave guides. This concept is illustrated also in Figure 2, where a phosphor based optical array relays out of the vacuum the space-resolved spectra recorded by the TG instrument. The use of optical rather than electrical signal path in the vacuum vessel could have a better chance of withstanding the harsh burning plasma environment.

The increased immunity of the optical array design to noise and nuclear radiation in the tokamak environment is illustrated in Figure 7, which compares MHD fluctuations measured on NSTX with an USXR optical array having CsI (Tl) converter, to the same measurement performed using a conventional photodiode array [30]. Both the noise estimated to arise from neutron interactions and the electromagnetic pickup are much reduced in the optical device.

The first element of the in-vessel detector is a thin XUV phosphor. Phosphor converters have also been proposed for energy integrated X-ray imaging in ITER [31]. Our investigations of XUV inorganic phosphors have identified some good candidates as concerns their efficiency $[32,33]$. For instance, a $2 \mathrm{mg} / \mathrm{cm}^{2}\left(\approx 6 \mu \mathrm{m}\right.$ thick) $\mathrm{P} 45\left(\mathrm{Y}_{2} \mathrm{O}_{2} \mathrm{~S}: \mathrm{Tb}\right)$ layer was found to emit about 60 visible photons in $4 \pi$ for a $525 \mathrm{eV}$ incident photon and about 3.6 visible photons for a $75 \mathrm{eV}$ photon [32]. The active research towards the development of directionally emitting, multilayered transparent phosphor films might lead to further efficiency improvements [34].

Detailed radiation transport calculations are needed to assess the signal to nuclear background ratio for each particular device and geometry. For an order of magnitude estimate one can nevertheless compare the XUV power incident on a detector 'pixel', to the ITER absorbed dose predictions. For instance, for the TG spectrometer a few meters away from the divertor, one can assume the radiation field at the location of the ITER secondary mirrors $\left(\approx 310^{9} \mathrm{~cm}^{-2} \mathrm{~s}^{-1}\right.$ energetic neutron and gamma flux, producing $\approx 10^{-2} \mathrm{~Gy} / \mathrm{s}$ absorbed dose rate in silicon [1]). Similarly, for the MLM arrays operating at a shorter distance (e.g., $\approx 2 \mathrm{~m}$ ) from the plasma, one may assume as nuclear background the geometric average between the first and secondary ITER mirror dose rates $\left(\approx 510^{-2} \mathrm{~Gy} / \mathrm{s}\right)$.

Using these values and scaling the radiation interaction coefficients from Si to the P45 composition, one obtains an absorbed power of $\approx 0.2 \mathrm{nW}$ for the $120 \mu \mathrm{m} \times 6 \mathrm{~mm}$ pixel of the TG device and $\approx 12 \mathrm{nW}$ for the $3 \times 3 \mathrm{~mm}$ MLM monochromator detector, respectively. This indicates that both instruments could measure the lower end of their respective brightness ranges $\left(10^{15}\right.$ photons $\mathrm{cm}^{-2} \mathrm{sr}^{-1} \mathrm{~s}^{-1}$ for the divertor and $10^{13} \mathrm{~cm}^{-2} \mathrm{sr}^{-1} \mathrm{~s}^{-1}$ for the main plasma) with around unity signal-to-background ratio.

The phosphor would be deposited on a thin transparent substrate, such as for instance a fiber optic plate. Nuclear radiation induced transmission loss and luminescence is an issue for thick windows [1]. However, ITER research has lead to the development of glass having improved radiation resistance, such as hydrogen hardened KU-1 [35, 36]. Assuming the use of 
such glass, the visible light attenuation in a $1 \mathrm{~mm}$ thick plate would be negligible $(\leq$ a few percent after $510^{17} \mathrm{~cm}^{-2} \mathrm{E}>0.1 \mathrm{MeV}$ neutrons [36]).

A more significant effect could have secondary electrons generated in the glass substrate by the energetic gamma flux. Assuming again ITER conditions, the flux of $\approx 310^{9} \mathrm{~cm}^{-2} \mathrm{~s}^{-1} \mathrm{MeV}$ gamma rays at the secondary mirror position would translate into $\approx 10^{5} \mathrm{~s}^{-1}$ Compton electrons of few hundred keV average energy impacting the $120 \mu \mathrm{m}$ x $6 \mathrm{~mm}$ pixel of the TG instrument. Using the stopping power approximation, the energy deposited in the $\approx 2 \mathrm{mg} / \mathrm{cm}^{2}$ phosphor layer would then be $\approx 0.1 \mathrm{nW}$, which is also comparable to the lower range of XUV signals expected from the divertor. A similar conclusion obtains for the detector of the MLM device.

Finally, since during the discharge the phosphor would be immersed in a low pressure D$\mathrm{T}$ mixture, it is of interest to evaluate the potential effect of tritium beta decays. The beta emission rate at e.g., $10^{-5}$ torr tritium pressure is $\approx 210^{3} \mathrm{~cm}^{-3} \mathrm{~s}^{-1}$, resulting in a few hundred beta electrons incident on the TG pixel from $\mathrm{a} \approx 10 \mathrm{x} 10 \mathrm{x} 10 \mathrm{~cm}^{3}$ spectrometer volume. With $\approx 5.7 \mathrm{keV}$ mean beta energy, the absorbed power in the TG phosphor pixel is negligible (pW range). While tritium retention in the phosphor might increase this value, results from spectrometers equipped with phosphor converters and exposed to tritium suggest that the effect is not large [37].

In conclusion, a few $\mu \mathrm{m}$ thick phosphor would be 'transparent' to energetic neutrons, gamma and secondary electrons, enabling in principle the detection of quite small XUV signals inside the burning plasma vessel. The effects of gamma and neutron irradiation on efficient XUV converters need however to be investigated. The research so far focused on X-ray and gamma scintillators [38]. While irradiation tests of rare-earth phosphors with $\mathrm{MeV}$ protons have identified some radiation hard candidates, it is not clear whether these results can be extrapolated to energetic neutron and gamma irradiation (see Ref. 31 and references therein).

\section{IV.2 Visible signal amplification}

Assuming an efficient converter such as P45, the above levels of XUV power incident on the phosphor would translate into a large number of converted visible photons (few $10^{8}$ to $10^{10} \mathrm{~s}^{-1}$ per pixel, or detection channel). At the lower end of this range it would be desirable to boost the light intensity before transport to the outside world. One type of device that could accomplish this task is the sealed proximity focused image intensifier. Due to the combined gain of the multichannel plate (MCP) and proximity focused phosphor these devices can achieve light amplification up to $\approx 10^{4} \mathrm{~W} / \mathrm{W}[7]$.

In neutron and gamma fluxes $\leq$ several $10^{8} \mathrm{n} / \mathrm{cm}^{2} \mathrm{~s}^{-1}$ the noise from conventional MCP intensifiers is low [7]. In stronger fluxes the noise becomes a problem, as indicated by spectrometers operated during high power D-T experiments at TFTR and JET (neutron and gamma fluxes up to a few $10^{12} \mathrm{~cm}^{-2} \mathrm{~s}^{-1}$ ) $[37,39,40]$. The main source of intensifier noise was estimated to be gamma interactions in the MCP [40]. 
This might not be surprising, as conventional MCPs are manufactured in lead doped glass having around $50 \%$ by mass lead content [41]. Since in addition the glass volume significantly exceeds that of the amplifying channels, this makes the MCP also quite sensitive to hard X-rays and gamma rays (e.g., $\approx 2 \%$ detection efficiency at $500 \mathrm{keV} \mathrm{[41]).}$

Advances in nanotechnology lead however to the development of new types of MCPs, likely much more 'transparent' to nuclear radiation than the conventional ones. These are Silicon-MCPs, or quartz-MCPs, manufactured by directly micro-machining the MCP pores in thin (tens of $\mu \mathrm{m}$ ) silicon wafers, which can eventually be oxidized to quartz $[42,43]$. Although at a developmental stage, devices having ultra-thin $(\approx 1 \mu \mathrm{m})$ walls, open volume in excess of $90 \%$, and high gain have been demonstrated. At the same time, the Si-MCPs are estimated to be more radiation resistant and insensitive to strong magnetic fields [42,43].

For the stronger signals generated by focusing instruments such as the MLM monochromator, proximity focused intensifiers consisting just of a photocathode and an electron phosphor would provide simple and radiation hard amplifiers. Finally, a new type of light amplifier of potential interest for the burning plasma could be the gaseous image intensifier [44]. This consists of an image intensifier in which the amplifying element is a Gaseous Electron Multiplier, or GEM [45]. While the Kapton foil of the conventional GEM is not radiation resistant and typically requires flowing the working gas, sealed GEM amplifiers based on silica capillary plates are currently being investigated [46].

In conclusion, the recent advances in nanofabrication might have opened also new possibilities for radiation resistant light amplifiers, which could be used to boost the visible light signal by $\approx 10^{2}-10^{4} \mathrm{~W} / \mathrm{W}$.

\section{IV.3 Visible signal transport}

The main obstacle towards the development of in-vessel optical diagnostics for the burning plasma might have been that the extraction of visible light signals using conventional, solid core optical fibers is not possible. As shown by many irradiation tests as well as by tokamak D-T operation, the transmission of solid core fibers significantly degrades at the radiation levels expected near the burning plasma [1, 28, 40]. Although as mentioned, the recently developed hydrogen hardened fibers have improved radiation resistance, their use in ITER is presently limited to outside the vacuum vessel [1, 28]. Finally, even if the transmission problem could be mitigated, a major obstacle remains the radiation induced luminescence in the fiber core, which over long path lengths can give raise to large background levels $[1,36,40]$.

It was earlier suggested that hollow optical fibers might alleviate these problems [40]. The physical concept and technology for the production of efficient hollow fibers was not available at that time however. Recent advances in nanotechnology have changed this situation as well. Thus, presently a revolution is underway in the fiber optic technology, with the advent of 
'photonic-crystal' hollow fibers $[47,48]$. These carry light based on a completely different physical principle than solid core fibers. Instead of total internal reflection on an outer cladding and transmission of the reflected light through a solid core, the wave is guided by constructive interference on a layer of sub-wavelength holes surrounding a larger hollow core, as illustrated in Figure 8. Another type of hollow fiber based on diffraction on coaxial multilayers is the recently developed OmniGuide® Bragg fiber [49].

We advance that these types of fibers could offer a solution to the problem of visible light transport in the burning plasma environment. The reason being that in these fibers more than 95 $\%$ of the light energy can be guided through the hollow core, instead of solid silica. Thus, even if the glass darkens due to irradiation, only a negligible light attenuation along the microscopic distance between the guiding holes might have an impact on the overall transmission. In addition, very little of the fluorescence light generated in the thin silica walls should be guided through the core. In conclusion, one can a priori expect that the 'photonic crystal' fibers will perform well up to high levels of irradiation. Finally, like all interferential devices, the hollow fibers have a defined wavelength bandpass, which could be useful for the rejection of any parasitic light.

Presently the performance of these fibers at visible wavelengths is relatively modest (numerical apertures around 0.12 , core diameters of $\leq 15 \mu \mathrm{m}$ and attenuation of the order of 0.8 $\mathrm{dB} / \mathrm{m}$ ), since they have been primarily optimized for the NIR wavelengths of interest in telecommunications. It is however in principle possible to improve these parameters and numerical apertures $\geq 0.3$ and diameters of a few hundred $\mu \mathrm{m}$ are considered technically feasible [50]. Conversely, XUV-NIR phosphors could be explored, in order to match the highly efficient NIR hollow fibers.

To conclude, combining efficient XUV converters with radiation hard light amplifiers and hollow waveguides it might be possible to develop in-vessel detectors for burning plasma experiments. For instance, assuming $1 \% \mathrm{XUV}$ conversion efficiency, $10^{3} \mathrm{~W} / \mathrm{W}$ light amplification and $\approx 1 \%$ transmission for the hollow fiber bundles the number of visible photons counted outside the vacuum for the TG device would be in the $10^{7}-10^{9} \mathrm{~s}^{-1}$ range. 


\section{CONCLUSIONS}

Recent advances in nanofabrication and technology may enable manipulating and detecting light in novel ways, better suited to the burning plasma environment than in conventional spectroscopic instruments. For instance, as advanced in the present paper, light extraction using diffractive optical elements might be more reliable than using reflective mirrors. Further on, transporting visible light signals with hollow wave guides such as the "photonic crystal' fiber, might be more immune to nuclear radiation and electromagnetic perturbations than transporting low electrical signals.

Many more possibilities than those suggested in our paper might exist of combining these new optical elements into robust, radiation hard and lower cost diagnostics for the burning plasma. Given the importance of the successful demonstration of a reliable and cost effective fusion reactor, it might be essential to investigate also such alternative diagnostic possibilities.

\section{ACKNOWLEDGEMENTS}

The present work is supported by US Department of Energy grant no. DE-FG02-99ER54523.

This work was performed under the auspices of the U. S. Department of Energy by University of California, Lawrence Livermore National Laboratory under contract No. W-7405-Eng-48. 


\section{REFERENC ES}

1. A.E. Costley, D.J. Campbell, S. Kasai, K.E. Young, V. Zaveriaev, Fus. Eng. Des, 55, 331 (2001)

2. V. Voitsenya et al., Rev. Sci. Instrum. 72, 475 (2001)

3. K.Yu. Vukolov, M. I. Guseva, S.A. Evstigneev, L. S. Danelyan, V. M. Gureev, A.A. Medvedev, S.N. Zvonkov, 30th EPS Conference on Contr. Fusion and Plasma Phys., St. Petersburg, 7-11 July 2003 ECA Vol. 27A, P-2.76

4. A. Malaquias, M. von Hellermann, P. Lotte, S.Tugarinov, V.S.Voitsenya, 30th EPS Conference on Contr. Fusion and Plasma Phys., St. Petersburg, 7-11 July 2003 ECA Vol. 27A, O-3.4C

5. T. Wilhein, S. Rehbein, D. Hambach, M. Berglund, L. Rymell, H.M. Hertz, Rev. Sci. Instrum. 70, 1694 (1999)

6. C. Brinkman et al., Proc. SPIE Vol. 4012, p. 81-90, X-Ray Optics, Instruments, and Missions III; J. E. Truemper, B. Aschenbach Editors., July 2000

7. B. Blagojevic, D. Stutman, M. Finkenthal, H. W. Moos, R. Kaita, and R. Majeski, Rev. Sci. Instrum. 74, 1988 (2003)

8. N. C. Hawkes et al., in Diagnostic For Experimental Fusion Reactors 2, Edited by Stott et al., Plenum press, New York 1988, p. 297

9. M. Mayer, R. Behrisch, C. Gowers, P. Andrew, A. T. Peacock., in Diagnostic For Experimental Fusion Reactors 2, Edited by Stott et al., Plenum press, New York 1988, p. 279

10. A.S. Kukushkin, H. D. Pacher, D. Coster, G. W. Pacher, D. Reiter 30th EPS Conference on Contr. Fusion and Plasma Phys., St. Petersburg, 7-11 July 2003 ECA Vol. 27A, P-3.195

11. P. J. Caldwell, E. T. Arakawa, T. A. Callcott, Appl. Optics 20, 3047 (1981) 
12. S.A. Flodstrom and R.Z. Bachrach, Rev, Sci. Instrum. 47, 1464 (1976)

13. N. M. Ceglio, A. M. Hawryluk, D. G. Stearns, M. Kühne, P. Müller, Optics Lett. 13, 267 (1988)

14. G. R. Morrison, in X-ray Science and Technology, G. Michette and C. J. Buckley Editors, IOP Publishing, 1993. p. 319.

15. L. Kipp, M. Skibowski, R. L. Johnson, R. Berndt, R. Adelung, S. Harm, R. Seemann, Nature 414, 184 (2001)

16. K. Kincade, Laser Focus World, February 2004

17. Jon M. Bendickson, Elias N. Glytsis, and Thomas K. Gaylord, J. Opt. Soc. Am. A 16, 113 (1999)

18. B. L. Henke, E. M. Gullikson, J. Kerner, A. L. Oren and R. L. Blake, Journal of X-ray Science and Technology 2, 17 (1990)

19. S. P. Regan, K. B. Fournier, M. J. May, V. Soukhanovskii, M. Finkenthal, and H. W. Moos, Rev. Sci. Instrum. 68, 1002 (1997)

20. D. Stutman, M. Finkenthal, V. Soukhanovskii, M. J. May, H. W. Moos, and R. Kaita, Rev. Sci. Instrum. 70, 572 (1999)

21. V. A. Soukhanovskii, D. Stutman, M. Iovea, M. Finkenthal, H. W. Moos, T. Munsat, B. Jones, D. Hoffman, R. Kaita, and R. Majeski, Rev. Sci. Instrum. 72, 737 (2001)

22. D. Stutman, M. Finkenthal, L. Delgado-Aparicio, K. Tritz , N. Tamura, D. Kalinina, Proceedings of the $15^{\text {th }}$ Topical Conference on High Temperature Plasma Diagnostic, SanDiego, 2004, submitted to Review of Scientific Instruments

23. L. A. Shmaenok, S. V. Golovkin, V. N. Govorun, A. V. Ekimov, N. N. Salashchenko, V. V. Pickalov, V. P. Belik, F. C. Schuller, A. J. H. Donne, A. A. M. Oomens, K. A. Prokhorov, S. S. Andreev, A. A. Sorokin, B. G. Podlaskin, L. V. Khasanov, Rev. Sci. Instrum. 72, 1411 (2001)

24. D. Stutman, M. Finkenthal, M. Iovea, V. Soukhanovskii, M. J. May, and H. W. Moos, Rev. Sci. Instrum. 72, 732 (2001) 
25. S. Tugarinov, A. Krasilnikov, V. Dokouka, R. Khayrutdinov, I. Beigman, I. Tolstikhina, L. Vainshtein, M. von Hellermann, A. Malaquias, Rev. Sci. Instrum. 74, 2075(2003)

26. V. A. Soukhanovskii, D. Stutman, M. Finkenthal, H. W. Moos, R. Kaita, and R. Majeski, Rev. Sci. Instrum. 72, 3270 (2001)

27. S.P. Regan, M.J. May, V. Soukhanovskii, M. Finkenthal, and H.W. Moos, E.H. Farnum, F.W. Clinard, Jr., C. Tarrio and R. Watts, Rev. Sci. Instrum., 68, 757 (1997).

28. S. Yamamoto et al., J. Nucl. Mat. 283, 60 (2000)

29. F. H. Seguin, R. D. Petrasso and C.-K. Li, Rev. Sci. Instrum. 68, 753 (1997)

30. L. F. Delgado-Aparicio, D. Stutman, K. Tritz and M. Finkenthal, R. Kaita, L. Roquemore, D. Johnson and R. Majeski, Proceedings of the $15^{\text {th }}$ Topical Conference on High Temperature Plasma Diagnostic, San-Diego, 2004, to appear in Review of Scientific Instruments

31. Zurro, B., Burgos, C., Ibarra, A. and McCarthy K. J, Fusion Eng. Des. 34/35, 353 (1997)

32. S.P. Regan, L.K. Huang, M.J. May, H.W, Moos, D. Stutman, S. Kovnovich and M. Finkenthal, Appl. Optics 33, 3595 (1994)

33. V. A. Soukhanovskii, S. P. Regan, M. J. May, M. Finkenthal and H. W. Moos Rev. Sci. Instrum. 74, 4331 (2003)

34. See e.g. , www.esrf.fr/Conferences/UsersMeeting2003/Detectors/Speakers/roger_durst.pdf

35. K.Yu. Vukolov, B.A. Levin, Fusion Eng. Des. 66-68, 861 (2003)

36. B. Brichard, A. Fernandez Fernandez, H. Ooms, F. Berghmans, M. Decréton, A. Tomashuk, S. Klyamkin, M. Zabezhailov, I. Nikolin, V. Bogatyrjov et al., Journal of Nuclear Materials, In Press, Corrected Proof, Available online 17 June 2004,

37. I.H. Coffey, R. Barnsley, Proceedings of the $15^{\text {th }}$ Topical Conference on High Temperature Plasma Diagnostic, San-Diego, 2004, to appear in Review of Scientific Instruments 
38. A.S. Tremsin, J.F. Pearson, A.P. Nichols, A. Owens_, A.N. Brunton, G.W. Fraser, Nuclear Instruments and Methods in Physics Research A 459, 543 (2001)

39. K. W. Hill, H. Adler, M. Bitter, E. Fredrickson, S. von Goeler, 1-1. Hsuan, A. Janos, D. Johnson, A. T. Ramsey, and G. Renda, Rev. Sci. Instrum. 66, 913 (1995)

40. A. Ramsey, Rev. Sci. Instrum. 66, 873 (1995)

41. J.L. Wiza, Nucl. Instr. Meth. 162, 587 (1979)

42. A. Tremsin, J. V. Vallerga, O. H.W. Siegmund, C. P. Beetz and R. W. Boerstler Proc. SPIE, vol. 4854, "Future EUV-UV and Visible Space Astrophysics Missions and Instrumentation", Hawaii (2002)

43. C. P. Beetz, R. Boerstler, J. Steinbeck, B. Lemieux, D. R. Winn, Nuclear Instruments and Methods in Physics Research A 442, 443 (2000)

44. D. Mörmann, M. Balcerzyk, A. Breskin, R. Chechik, B. K. Singh and A. Buzulutskov Nucl. Instr. Meth. A 504, 93(2003)

45. F. Sauli, Nucl. Instr. and Meth. A 386, 531(1997).

46. C. Iacobaeus, A. Breskin , M. Danielsson, T. Francke, D. Mörmann , J. Ostling and V. Peskov, Nuclear Instruments and Methods in Physics Research Section A: In Press, Corrected Proof, Available online 13 April 2004,

47. C. M. Smith, N. Venkataraman, M. T. Gallagher, D. Müller, J. A. West, N. F. Borrelli, D. C. Allen, and K. W. Koch, Nature 424, 657 (2003)

48. J.D. Joannopoulos, P.R. Villenueve, S. Fan, Nature 386 (1997) 143.

49. M. Ibanescu, Y. Fink, S. Fan, E. L. Thomas, J. D. Joannopoulos, Science 289, 415 (2000)

50. R. Kristiansen, Crystal-Fibre Inc., private communication 